Meta

Journal des traducteurs

Translators' Journal

\title{
La traduction juridique : art ou technique d'interprétation?
}

\section{Jean-Claude Gémar}

Volume 33, numéro 2, juin 1988

L'enseignement de la traduction au Canada - Teaching Translation

in Canada

URI : https://id.erudit.org/iderudit/002850ar

DOI : https://doi.org/10.7202/002850ar

Aller au sommaire du numéro

Éditeur(s)

Les Presses de l'Université de Montréal

ISSN

0026-0452 (imprimé)

1492-1421 (numérique)

Découvrir la revue

Citer cet article

Gémar, J.-C. (1988). La traduction juridique : art ou technique d'interprétation? Meta, 33(2), 304-318. https://doi.org/10.7202/002850ar d'utilisation que vous pouvez consulter en ligne.

https://apropos.erudit.org/fr/usagers/politique-dutilisation/ 


\section{LA TRADUCTION JURIDIQUE : ART OU TECHNIQUE D'INTERPRÉTATION ?*}

JEAN-Claude GÉmar

Université de Montréal, Montréal, Québec

Pour postuler que l'on peut enseigner à traduire des textes de nature juridique il faut d'abord partir du principe que traduire est possible. Pour cela, il est nécessaire d'écarter les arguments, qui ne manquent ni de force ni de cohérence, de certains théoriciens, entre autres, de la linguistique pour lesquels "l'identité " — soit l'égalité parfaite des mathématiciens - des mots et, bien entendu, des notions qu'ils représentent est impossible à atteindre lorsque l'on veut passer d'un code linguistique à un autre.

À la place de l'identité, notion mathématique et abstraite, nous proposons le principe de l'équivalence (des situations), donc des moyens particuliers dont dispose chaque code linguistique pour décrire celles-ci, qu'elle soit "dynamique ", "fonctionnelle " ou autre. Nous retiendrons pour notre part l'idée d'équivalence fonctionnelle comme caractéristique de l'activité traduisante appliquée au domaine juridique'.

Ensuite, il faut postuler que le « juridique " peut aussi être traduit, ce qui pose un tout autre problème, d'ordre épistémologique essentiellement, sur lequel nous nous sommes déjà expliqué ${ }^{2}$, qui est loin d'avoir été résolu par les juristes eux-mêmes et ne le sera peut-être jamais, le droit n'étant pas, quoi qu'en pensent d'aucuns, une science exacte mais une discipline sociale.

Les nombreuses années que nous avons consacrées à enseigner la traduction juridique - ou, plutôt, LES traductions juridiques, ainsi que nous le verrons - comme discipline autonome nous ont intimement convaincu que, d'une part, on peut traduire des textes partiellement ou entièrement juridiques et que, d'autre part, il est possible d'enseigner à les traduire correctement, aux conditions qui seront énoncées un peu plus loin. En effet, nul ne saurait songer sérieusement à aborder cet enseignement de la même manière qu'une autre activité de traduction (littéraire, générale, technique...), car le texte juridique - loi, règlement, jugement, contrat... - est le plus souvent porteur d'effets susceptibles de mettre en ceuvre une responsabilité quelconque, une obligation de faire ou de ne pas faire. Le caractère contraignant ${ }^{3}$ du texte juridique le distingue généralement des autres catégories de textes. Cette contrainte s'exerce plus particulièrement dans un pays comme le Canada où cohabitent deux langues officielles, deux systèmes juridiques fort différents, donc également deux systèmes d'interprétation du droit, soit dleux conceptions, parfois opposées, de la manière de " rechercher l'intention [du législateur] en tenant compte du texte, du contexte, de la finalité, de l'histoire...4 ". Ces conditions singulières marquent clairement la spécificité de l'activité traduisante en cette matière et en conditionnent l'enseignement.

Le droit, système complexe d'organisation des sociétés 5 , est un des rares secteurs de l'activité humaine qui prévoit ses propres règles d'interprétation des textes qu'il sécrète. Le système juridique est ainsi fait qu'il ne laisse à personne d'autre que les juristes, des juges pour la plupart, le soin de " dire le droit ", c'est-à-dire ici de fixer le sens " officiel " à attribuer obligatoirement à un mot, un terme, une disposition (ou clause, selon le cas), un principe, voire à un texte entier, lorsque ce sens prête à diverses interprétations 
possibles par suite de circonstances particulières, dont le silence, l'obscurité, l'ambiguïté, l'imprécision, la maladresse, etc.

Il s'ensuit qu'enseigner à traduire des textes juridiques revient, pour l'essentiel, à apprendre à interpréter - au sens fort de l'anglais to construe - correctement le sens d'un texte, non seulement à trouver le sens des mots, opération somme toute normale voire banale ${ }^{6}$, et parfois fallacieuse ${ }^{7}$, mais encore et surtout à retrouver le sens que l'auteur (législateur, juge, rédacteur en général...) a réellement voulu donner à son texte. Pour ce faire, le traducteur doit savoir « lire », au sens plein du mot. En paraphrasant un traducteur célèbre, André Maurois, nous postulerons ceci : écrire, bien écrire, est difficile ; lire, bien lire, plus difficile encore. Cette responsabilité incombe au traducteur en général et au traducteur juridique en particulier. Former des traducteurs de manière qu'ils puissent atteindre un tel objectif - interpréter correctement le sens du texte à traduire, c'est-à-dire du message dont il est porteur - constitue un devoir pour le professeur de traduction, quel que soit son domaine d'intervention.

Aussi, avant de présenter une quelconque formule d'enseignement établie en fonction d'un public cible donné et présumément générale ${ }^{8}$, il nous a semblé judicieux d'énoncer les principes fondamentaux - la méthode pédagogique ou philosophie personnelle - qui guident notre action pédagogique et d'où procède notre enseignement. Cela, afin de mieux comprendre comment cette responsabilité, celle du traducteur comme celle du professeur, peut être intégrée dans la formation du traducteur et de quelle manière celui-ci pourrait l'assumer avec confiance. Il importe donc, au risque de prêcher dans le désert, de dénoncer quatre idées reçues en traduction juridique, stéréotypes généralement véhiculés par les juristes et largement acceptés par les traducteurs, qui n'en peuvent mais.

La première, la plus importante par sa portée, la plus grave quant à ses incidences, porterait à croire que seul un juriste peut faire de la traduction juridique. La deuxième est qu'en la matière le " juridique » a toujours préséance sur la " traduction ", que le fond prime la forme. Selon la troisième, l'on ne peut enseigner à traduire dans le domaine du droit à des profanes et, à supposer que l'on y parvienne, le traducteur est tenu de s'effacer devant la compétence du juriste superviseur, lequel connaît le droit alors que le traducteur l'ignore. La quatrième, enfin, pose la question de la place de la terminologie des mots - dans le langage du droit et la traduction juridique.

Les éléments de réponse apportés à ces quatre idées reçues, fausses dans la plupart des cas, constituent à notre sens les principes de base de la formation du traducteur de textes juridiques et conditionnent tout discours sur l'enseignement de la traduction juridique. Ils s'inspirent de principes méthodologiques découlant de l'expérience et de faits précis de traduction, de solides convictions aussi, fondées sur des données empiriques pour la plupart et des observations ${ }^{9}$, et non sur la seule intuition du chercheur praticien - le "pédagotrad" cher à J.-P. Vinay — que nous croyons être.

\section{LE JURISTE, TRADUCTEUR "NATUREL"}

L'idée que le juriste ${ }^{10}$ est la personne tout indiquée pour traduire des textes juridiques est universellement répandue. Nous prétendons, quant à nous, qu'un juriste qui ne serait pas formé à la traduction, à ses méthodes, à ses contraintes n'est pas plus qualifié pour traduire qu'une secrétaire d'entreprise, un comptable, un architecte, un médecin ou un plombier. Or, particulièrement au Canada, l'on considère qu'un avocat (ou une personne détenant un diplôme de droit), dans la mesure où il possède quelques rudiments de langue seconde ou troisième, est habilité ex officio à traduire des textes de nature juridique.

Nous ne pouvons souscrire à cette opinion. Le fait de connaître un domaine ne confêre d'aucune manière les compétences très particulières qu'exige l'activité tradui- 
sante, même pour qui possède assez bien une ou plusieurs langues étrangères. À l'évidence, il est de brillantes exceptions, comme il s'en trouve dans tous les domaines, mais de quelques cas isolés on ne saurait tirer une loi, et pour quelques réussites brillantes, combien d'échecs navrants ? C'est que, au-delà de la " simple » connaissance du droit, il y a celle, essentielle, de la langue, de ses nuances et subtilités, entre autres terminologiques mais aussi (et surtout) syntaxiques et stylistiques. Cet apprentissage, sauf très rare exception, ne fait pas partie des enseignements dispensés dans les facultés de droit nordaméricaines ou autres, situation qui suscite chez certains juristes de vives critiques. Ils souhaiteraient voir mettre davantage l'accent sur les techniques de rédaction et la correction linguistique minimale dans la formation générale des étudiants en droit, en pays de langue anglaise ${ }^{11}$ comme en pays francophone $e^{12}$, pour combler une lacune enracinée dans $l^{\prime}$ histoire et découlant de siècles de traditions juridiques ${ }^{13}:$ remedies precede rights (la procédure prime le droit). De fait, si l'on en croit l'Encyclopaedia Britannica, ce principe cardinal expliquerait la différence des démarches entre common lawyers et civilistes :

The main difference between the systems consists of the ways in which the norms of the law are articulated and in which new rules are derived from older ones in novel cases. (...) In the common law, [the] role of adapting the law to changing conditions has traditionally been the task of the judges. In civil-law countries, the task had generally been performed by university professors... (15th ed., Macropaedia, vol. 4, 1978, p. 666, Vo Civil Law.)

En vertu de ce principe, l'opinion d'un auteur ne pouvait prévaloir sur celle d'un juge exprimée dans une décision et, surtout, un arrêt ${ }^{14}$. Leur fonction étant assez peu valorisée dans un tel système, les auteurs (la doctrine) ont généralement été peu tentés de rédiger de vastes traités savants pour analyser, commenter ou expliquer le droit. L'absence de longue tradition en matière de rédaction d'ouvrages savants est notoire en common law nord-américaine - quoique moins accentuée aux États-Unis qu'au Canada - et a considérablement influencé le droit et les juristes civilistes.

Toutefois, par-delà les situations particulières, il reste que le langage du droit pose un problème universel, toutes langues et tous systèmes confondus, comme en témoignent les innombrables critiques et satires, aussi drôles qu'imaginatives, dont les hommes de loi et leur langage sont accablés dans la plupart des cultures. La littérature universelle en regorge. Dans le domaine français seulement, il suffira de citer Rabelais, Montaigne, Voltaire et Montesquieu, celui des Lettres persanes; un juriste pourtant ! Ces critiques sont constantes, depuis les Grecs et les Romains jusqu'à nos jours. Elles ne peuvent être totalement dénuées de fondement. Ces fondements transparaissent clairernent dans la plupart des textes juridiques rédigés ou traduits en français, et pas seulement parce que l'influence des techniques de rédaction en common law s'y fait sentir, mais plutôt en raison d'une formation linguistique insuffisante de la part du rédacteur, qu'il soit juriste pur ou juriste traducteur. Ce fait est indéniable : on apprend à rédiger, tout comme on apprend à traduire.

Pour conclure la première partie de cette étude nous formulerons deux recommandations. La première est adressée aux juristes désirant pratiquer la traduction, même et surtout juridique ; la seconde est destinée aux traducteurs qui désirent exercer leur activité dans le domaine du droit.

Aux premiers, nous conseillerons de se former à l'art de traduire ${ }^{15}$ et, avant tout, de rédiger. Aux seconds, nous ne pouvons que recommander fortement d'apprendre le droit afin de mieux maîtriser leur art en l'édifiant sur des bases juridiques, donc " techniques ", solides. Moyennant quoi, nous ne doutons pas que les uns et les autres parvien- 
nent à des résultats satisfaisants pour eux, certes, mais aussi (surtout ?) pour l'ensemble de la collectivité.

\section{DROIT ET TRADUCTION : UN CONFLIT D'INTÉRÊTS ?}

Lorsque nous avançions un peu plus haut que le traducteur a la responsabilité de traduire - le mieux possible, il va sans dire, — nous entendions par là qu'il a, envers la langue d'arrivée, une responsabilité particulière : exprimer le contenu (ou message) du texte de départ dans une langue claire, précise et simple, soit le français pour ce qui nous concerne. Pour dégager le principe de la responsabilité du traducteur juridique, nous retiendrons la distinction que font les juristes entre obligation de moyens, obligation de résultat et obligation de garantie 16 .

En premier lieu, le traducteur ${ }^{17}$ a une obligation de moyens : il dispose des ressources quasi illimitées que possède toute langue et dont il doit tirer le meilleur parti, et non simplement un parti honnête ou convenable. Si, en théorie, il importe relativement peu, quant aux effets éventuels de sa traduction, que le traducteur emploie " en vertu de » plutôt que « aux termes de " ou " conformément à ", " malgré » au lieu de "nonobstant ", en pratique l'abus du même terme, ses trop nombreuses répétitions auront un effet stylistique déplorable pouvant aller jusqu'à indisposer le lecteur ou l'usager du texte. En revanche, traduire (en matière de contrat) cancellation par "cancellation " au lieu d'annulation (ou, selon le cas : rescision, résiliation, résolution, révocation) du contrat constitue une erreur à la fois linguistique et juridique ${ }^{18}$ révélant chez celui qui la commet une méconnaissance coupable sur deux plans, celui de la langue et celui du droit.

Cette obligation de moyens est particulièrement contraignante dans un contexte sociopolitique et linguistique aussi sensible que peut l'être celui du Canada, particulièrement au Québec, dans le secteur public comme dans le privé. Les efforts considérables des jurilinguistes canadiens depuis une quinzaine d'années ont porté des fruits et une de leurs moindres réussites n'est pas d'être parvenus à imposer un haut niveau de qualité de la langue juridique française dans les textes officiels du gouvernement fédéral, généralement traduits de l'anglais au français (lois et règlements, décisions des tribunaux....), comme dans ceux du Québec (rédaction des lois et des règlements, traduction en anglais des codes civils...). Cet acquis est réel. Il a été atteint à force de volonté, de courage et d'acharnement et n'en revêt que plus de valeur symbolique. En conséquence, la responsabilité du traducteur juridique envers son texte n'en est que plus grande.

C'est l'exercice libre et entier de cette responsabilité qui l'a conduit à oser, par exemple, dans le Privacy $A c t^{19}$, à l'article 70 , paragraphe 3 , à intégrer dans l'alinéa b français, et dans une seule phrase, les deux subdivisions prévues dans le texte anglais. Quel meilleur exemple de traduction conforme à l'esprit et aux contraintes de la rédaction française pourrait-on proposer quand on sait avec quelle servilité les lois anglaises (statutes) ont longtemps été rendues en français, de peur de trahir le texte et, avant tout, le système de départ? Une telle liberté d'action révèle un degré d'autonomie rare que l'on souhaiterait trouver dans les traductions françaises des traités internationaux conclus au sein des Nations unies, de la C.E.E. (Communauté économique européenne) ou même de façon bilatérale entre un pays de langue française et un de langue anglaise.

Le fond du problème, toutefois, se situe ailleurs. Il est dans l'obligation de résultat que l'on attend du traducteur (juridique) et dans l'obligation de garantie, degré suprême de qualité ou fiabilité d'une traduction.

Il s'agira de s'entendre sur la portée du " résultat » recherché et de la "garantie » attendue. Il nous paraît évident (mais en sera-t-il de même pour la majorité des lecteurs ?) que la responsabilité du traducteur de lois, de jugements ou de traités internationaux ne se situe pas au même niveau - sur les plans professionnel, social, politique, éthique... - que celle de la personne qui traduit uniquement des contrats, des formules 
administratives ou commerciales à usage interne ou privé, des testaments ou des conventions collectives, sans vouloir en aucune façon minimiser les difficultés que présentent de tels textes.

Pour compléter cette esquisse de typologie, un troisième type de traductions sera celle des textes de doctrine. Quelle commune mesure y a-t-il entre les deux premiers et la personne qui traduit un livre de philosophie du droit ou un important traité de droit civil (ou pénal, constitutionnel ou autre) ? Reconnaissons sans fausse honte ce fait : en traduction comme en interprétation, il existe des niveaux de difficulté, de compétence et de qualité. En traduction juridique aussi. Le traducteur de ce troisième type de textes sera, plus encore que les deux autres, tenu d'effectuer une prestation triple : moyens + résultat + garantie.

Dans le premier cas, nous avons la puissance publique, l'État, qui s'exprime par la voie de textes formels, références obligées du justiciable, de l'administré, du citoyen. Dans le deuxième, nous sommes placés devant la multitude innombrable d'activités privées que secrète la société. Dans le troisième, nous avons affaire aux grands auteurs, les " autorités ", qui dissertent sur le droit, l'analysent, l'éclairent ou l'exposent, parfois à un degré d'abstraction propre aux grands esprits d'une époque donnée. Traduire les Blackstone, Pothier, Kelsen, Gény, Planiol et Ripert, Holmes, Carbonnier, etc. n'est pas à la portée du premier venu. Les moyens employés pour y parvenir importent, en l'occurrence, autant que le résultat, car toutes les nuances d'une pensée supérieure solidement structurée doivent être rendues. Enfin, la garantie du résultat assorti des moyens nécessaires vient parachever le tout.

Dans le premier cas, le traducteur œuvre dans un domaine que nous qualifierons de solennel, peut-être même de "sacré ", pour emprunter au vocabulaire liturgique. Estce que ce type de traduction, pour le juriste en tout cas, ne s'apparente pas d'une certaine façon à la traduction des textes sacrés ? La traduction d'une loi ne saurait être manipulée impunément ni traitée aisément. À l'instar de la rédaction des lois ${ }^{20}$, elle obéit à des règles très particulières que l'on ne transgresse pas sans risque. En cette matière, le traducteur juridique est tenu à la double obligation de résultat et de garantie et à une obligation $x$ de moyens.

Dans le deuxième cas, celui de la traduction juridique d'intérêt privé (par opposition à la traduction juridique d'intérêt public), l'obligation de moyens passe souvent au second plan. Le client, sauf rare exception, attend du traducteur qu'il rende avant tout le " contenu juridique » du texte de départ. Cette exigence (ou lacune, selon les points de vue : le plus souvent, ledit client ignore ce qu'il faut entendre par " contenu juridique ") est en grande partie responsable de la piètre qualité des textes juridiques courants que le commun des mortels a eu ou aura l'occasion de lire un jour ou l'autre chez son homme de loi, son assureur, vendeur d'automobiles ou de machines à laver. D'où l'impression, souvent justifiée, que les textes juridiques sont mal rédigés, bourrés d'impropriétés et de fautes en tous genres, pour ne rien dire du style. Ce faisant, en négligeant la question des rnoyens au profit du seul résultat, ce groupe contribue à déconsidérer le langage du droit aux yeux du public. L'obligation de garantie, elle aussi, passe au second plan, pour diverses raisons qu'il serait trop long de développer ici21.

Dans le troisième cas, enfin, traduire les grands auteurs de la doctrine revient le plus souvent à traduire des cuvres littéraires ou quasi littéraires. Le traducteur doit alors respecter davantage le texte de départ et son auteur, et sa prestation s'en ressentira. Son obligation sera triple, au degré le plus contraignant : rendre le " juridique " jusque dans les nuances les plus fines - qui se situent parfois à la pointe de la pensée ou de la recherche à un moment donné - mais en respectant les moyens employés par l'auteur, c'est-à-dire en s'efforçant de s'exprimer au même niveau, stylistique également. 
Sur ces deux points il est tenu à une obligation de qualité (= garantie) quant au fond et quant à la forme. En ce sens, on peut avancer que la traduction, en la matière, est d'essence supérieure et relève aussi en quelque sorte de l'intérêt général, car elle s'adresse à la communauté scientifique - le monde juridique - présente ou future, et, par voie de conséquence, à la collectivité entière.

Afin d'illustrer le principe de l'obligation triple du traducteur juridique, nous proposons le tableau suivant, non comme modèle absolu de la hiérarchie, subtile et fluctuante en réalité, des textes juridiques - ce qui pourrait laisser croire, à tort, à une quelconque fixité ou régularité des manifestations du droit -, mais plutôt comme exemple concret des diverses possibilités d'expression caractérisant le langage du droit.

Tableau des obligations du traducteur juridique*

\begin{tabular}{|c|c|c|c|c|}
\hline $\begin{array}{l}\text { Nature des } \\
\text { textes à } \\
\text { traduire }\end{array}$ & $\begin{array}{l}\text { Niveau de } \\
\text { difficulté } \\
\text { (1 à } 3 \text { ) }\end{array}$ & $\begin{array}{l}\text { Obligation } \\
\text { de } \\
\text { moyens }\end{array}$ & $\begin{array}{l}\text { Obligation } \\
\text { de } \\
\text { résultat }\end{array}$ & $\begin{array}{l}\text { Obligation } \\
\text { de } \\
\text { garantie }\end{array}$ \\
\hline
\end{tabular}

$\begin{array}{lllll}\text { Loi, } & 1 & + & + & +\end{array}$

Jugements et actes de procédure

$\pm \quad++\quad \pm$

Actes juridiques (contrat, 3 testament...)

$\pm \quad++\quad \pm$

$\begin{array}{lllll}\text { Doctrine } & 1 & ++ & ++\end{array}$

\footnotetext{
${ }^{*}$ Le signe + indique le degré de contrainte auquel est soumis le traducteur. Le signe \pm dénote un certain degré de latitude laissé au traducteur. Le niveau de difficulté des textes est indiqué de la façon suivante : $3=$ difficulté moyenne $; 2=$ difficile $; 1=$ très difficile.
}

Nous en arrivons maintenant à la troisième question préalable posée plus haut, celle de l'enseignement dispensé à un public de profanes. Selon une opinion largement répandue dans les milieux juridiques, l'on ne pourrait enseigner à traduire dans le domaine du droit à des profanes, sinon en pure perte.

\section{DES TÊTES " BIEN PLEINES " OU " BIEN FAITES " ?}

La querelle des Anciens et des Modernes ne sévit pas uniquement dans le domaine littéraire, on la retrouve aussi, entre autres, dans les facultés de droit. On connaît la controverse qui divise le monde du droit sur la formation des siens : vaut-il mieux former des «techniciens » du droit ou des " juristes "22, au sens noble et plein de ce terme, c'està-dire des personnes ayant une connaissance "éclairée " du droit, à la fois générale et approfondie, lato sensu plutôt que stricto sensu? 
La réponse à cette question dépendra sans doute du type de travail que l'on attend du juriste (ou du traducteur). Pour notre part, nous avons toujours opté pour le parti de former des traducteurs, entre autres, juridiques, qui aient la tête bien faite ${ }^{23}$.

Pour atteindre cet objectif, il ne suffit pas d'enseigner à traduire. Le professeur doit revêtir tour à tour, entre autres, la casquette du juriste, du comparatiste, du linguiste, de l'historien, du terminologue, du rédacteur et du documentaliste. Ajoutons à cela une solide base de culture générale, un minimum de logique, un peu de science politique, quelques rudiments de connaissances techniques et scientifiques, beaucoup de foi et d'enthousiasme pour motiver les étudiants, car (est-il besoin de le souligner ?) le sujet est vaste, retors et son étude parfois rébarbative pour le profane.

Ces réserves étant faites, une alternative fondamentale se présente d'emblée à qui veut enseigner la traduction juridique : former des spécialistes ${ }^{24}$, au sens technique du terme, à même de concurrencer les juristes sur leur propre terrain, ou s'efforcer plutôt d'inculquer des principes généraux, tenter de faire comprendre d'abord pour faire apprendre ensuite. L'option retenue en fonction des objectifs visés et des moyens et ressources dont dispose le formateur (public concerné, locaux, budget, horaire...) déterminera son activité pédagogique. Vouloir former des spécialistes, c'est demander à des non-juristes d'arriver en quelques séances à savoir "prendre la température" d'un texte ${ }^{25}$, c'est-à-dire à le saisir dans ses nuances les plus fines, en comprendre à la fois le sens et la portée, les présupposés (juridiques), les enjeux. D'autre part, cela revient également à viser la formation de traducteurs de haut niveau pouvant traduire, par exemple, des textes de loi et de doctrine.

Un tel objectif nous semble utopique, compte tenu du nombre moyen de cours et d'heures que l'on peut consacrer à cet enseignement dans un programme conventionnel, du niveau de départ de la grande majorité des étudiants - notamment au premier cycle - , des objectifs des divers programmes, etc. N'ayant aucunement cette prétention, nous avons choisi de former des "généralistes", pour traduire des textes de force et de difficulté moyennes, ce qui est déjà beaucoup. Il s'agira, en l'occurrence, d'amener l'étudiant au niveau suffisant pour qu'il puisse saisir, non la " température " du texte - degré supérieur de connaissance d'un domaine réservé au spécialiste et attendu, en droit, d'un juriste accompli - , mais en comprenne au moins le niveau, puisse savoir facilement le «temps" qu'il fait (beau ou mauvais, ensoleillé ou couvert, sec ou humide, venteux ou non...). Telle est la tâche du généraliste. Nous entendons par là une personne qui possède une bonne connaissance générale du droit et de ses divers domaines principaux, et donc de son vocabulaire général, soit le vocabulaire fondamental du droit. Celui-ci constitue la base de la connaissance juridique, qui se compose au minimum des obligations, des biens et de la famille, du droit public général, des institutions judiciaires, du droit pénal, du droit commercial et des différentes procédures, dont la civile et la pénale. Il1 n'est pas inutile non plus de connaître, à l'ère des voyages et des communications planétaires, le droit international — public et privé.

C'est beaucoup et peu à la fois. Beaucoup parce que le temps est compté, et vaste la matière. Peu quand on établit la comparaison - au fait, pourquoi comparer ? - entre la formation que reçoit un juriste et celle qui est donnée au traducteur, forcément lacunaire. D'où la nécessité de faire le plus avec le moins. En termes concrets, il importe, si l'on veut qu'il maîtrise les fondements de son art, de faire apprendre au futur traducteur juridique au moins les trois éléments du langage du droit que sont :

1. le corpus terminologique fondamental du droit, la nomenclature de quelque 1500 à 2000 termes de base de la langue juridique et les notions qu'ils recouvrent ; 
2. les techniques propres aux juristes - notamment documentaires - et les règles de citation des textes de référence (loi et règlement, décision, doctrine), avec leurs nombreuses abréviations souvent incompréhensibles pour le profane. En somme, ce que l'on enseigne dans les cours de méthodologie juridique ;

3. les formes particulières d'expression du droit, selon le type de textes visés : loi, jugement, contrat, commentaire d'arrêt, etc.

\section{DES MOTS ET DES CHOSES}

Le droit tiendrait-il seulement dans des mots ou est-il aussi, sinon davantage, un "langage »? Nombreux sont les juristes qui, à cause du mal polysémique dont souffre leur langue de spécialité ${ }^{26}$, partagent le point de vue de François Gény, pour lequel « la technique juridique aboutit, pour la plus grande part, à une question de terminologie ${ }^{27}$ ». Pour cette raison, on ne compte plus le nombre de traducteurs qui se sont vu reprocher de ne pas être davantage " fidèles " - euphémisme pour " servile " au texte de départ. On leur reprochait en réalité de manquer de précision dans les termes, c'est-à-dire de ne pas coller davantage aux mots de la langue de départ. Ce souci de précision fait honneur aux juristes. Il se conçoit parfaitement quand il s'agit de parler d'un "cautionnement " (somme d'argent) et non d'une "caution "; d'un " jugement ", et non d'un "verdict» (terme réservé au jury); d'une "résiliation" (de contrat) ou d'une " rescicion", "résolution ", etc., au lieu d'une " cancellation". Cette précision ou correction - des termes est nécessaire pour ne pas induire en erreur le lecteur ou la partie en cause. En droit, l'erreur peut avoir des conséquences incalculables : elle peut vicier un consentement et aboutir ainsi à l'annulation de l'acte.

Enfin, un terme technique présente un énorme avantage, il recouvre une réalité notionnelle précise, un champ sémantique donné et relativement stable qu'il serait difficile de contourner par une paraphrase ${ }^{28}$, par souci de clarté, de simplicité ou pour des raisons stylistiques.

Autre chose est d'employer, par exemple, un synonyme (locateur et bailleur, ayant droit et ayant cause, dolosif et frauduleux, contrat et convention...) à la place d'un terme. On sait que les juristes affectionnent les répétitions, au point d'en alourdir quelquefois leurs textes, et évitent soigneusement les changements synonymiques dans un même document. En dehors des formules figées par l'usage (" à ces causes", " en foi de quoi », "de plein droit »...), on comprend difficilement, la plupart du temps, le pourquoi et le comment de tels abus de langage, et l'on voit mal ce qui les justifie, alors que la société fournit de tels efforts pour rendre l'information plus accessible au plus grand nombre de gens en vertu du principe inapplicable : nul n'est censé ignorer la loi... Les tenants du plain language law (le droit exprimé en langage clair/simple), aux États-Unis, luttent contre d'énormes pesanteurs, préjugés et traditions mais n'en marquent pas moins régulièrement des points. L'exemple du Dictionnaire de droit prive ${ }^{29}$ devrait convaincre les plus sceptiques : le droit peut être exprimé en langage clair et (relativement) simple sans pour autant sacrifier à la précision et à la rigueur scientifique attendues d'un ouvrage de ce genre.

Nous en arrivons à la question fondamentale, qui tourne autour de la terminologie, certes, mais peut-être plus encore de la sémantique appliquée à la traduction et de la formulation du message ou rédaction. Placé devant un texte pragmatique - ce qu'est généralement un texte juridique - le traducteur doit-il se contenter de traduire les mots? Ou bien doit-il rendre le contenu, le message, avant tout ? Un traité de traductologie ne suffirait pas pour apporter une réponse objective à une telle question. Aussi, sans vouloir entrer dans une querelle de théoriciens, et quitte à choquer les - nombreux - juristes traditionalistes, nous avancerons que la langue a priorité sur le vocabulaire, 
le message sur les mots, le contenu sur le contenant, sans pour autant en conclure que le fond et la forme sont à dissocier ${ }^{30}$.

Nous voulons dire ceci : le traducteur, placé devant un terme ou une expression technique, devra-t-il toujours les rendre comme le ferait la machine à traduire programmée pour sortir un équivalent au terme de la langue de départ, un mot par un mot, à la façon d'un lexique bilingue offrant un équivalent français pour le substituer à un terme donné en anglais (ou à quelque langue que ce soit)? Beaucoup de personnes, dont de nombreux juristes, pensent encore que le traducteur a l'obligation de s'en tenir aux mots, de les agencer de telle sorte qu'ils produisent aussi une phrase ou un texte acceptables d'un point de vue linguistique. D'autres juristes, en nombre croissant, ouverts aux méthodes et techniques de la traduction, de la terminologie ou de la linguistique contemporaine, ne croient plus que le traducteur doive se plier impérativement à cette règle. Ils n'en pensent pas moins que le droit prime la langue. Une semblable attitude est compréhensible, quoique criticable, de la part de ceux qui considèrent que leur champ d'activité est unique et que leur discipline fonctionne de façon autarcique, abstraction faite de l'univers dans lequel elle se situe. Or, le droit est (que l'on me pardonne un tel truisme ?) dans la langue, et non le contraire, la langue dans le droit. D'ailleurs, s'il est un domaine éminemment social et linguistique, c'est bien celui du droit ! Du moment que le traducteur a rendu le message (juridique) de façon conforme aux normes, usages, servitudes et réalités linguistiques de la langue d'arrivée, il a rempli son obligation. Encore faut-il les connaître... Cela n'est pas, mais devrait l'être, la responsabilité des juristes. Pourtant, ce sont eux qui, le plus souvent, ont la haute main sur le texte final, le traducteur ayant rarement le dernier mot dans ce rapport inégal.

Deux exemples illustreront notre point de vue, l'un «terminologique ", pris hors contexte (dans un lexique, par exemple), l'autre "phraséologique", avec terminologie en contexte (celui d'une phrase courte).

\section{TERMS AND CONDITIONS (of a contract)} tions".

Équivalent français : les conditions (d'un contrat), et non "les termes et condi-

Explication : Du point de vue du droit civiliste, il est absurde de parler de "termes " et de " conditions". Ces deux mots s'imposent en anglais parce que la common law prévoit deux types de « conditions" (cf. René DAVID, les Contrats en droit anglais, 1973, Paris, L.G.D.J., par. 255, p. 240). Il s'ensuit que terms ne veut pas dire ici words (les mots), mais " condition 31 . Parler des " termes" du contrat constitue de ce fait, en français, un contresens (cf. Dictionnaire $d u$ droit privé, op. cit., Vo TERME, p. 183).

Il ne s'agit là toutefois, que d'un problème " terminologique " relativement simple, quoiqu'il porte sur une notion juridique fondamentale, celle des conditions du contrat. Prenons une phrase complète présentant plusieurs niveaux de difficultés (notamment, terminologiques, syntaxiques et stylistiques).

\section{Exemple phraséologique}

Adjudication implies the application of law to individual cases brought, through one means or another, before the bar of justice. [From : Oscar IBELE (1970): Political Science. An Introduction, Toronto, Chandler.]

Les termes techniques notables sont : adjudication, law, cases, bar (of justice). Une recherche élémentaire nous renseigne sur leur sens possible dans cette phrase : 
adjudication : The formal judgment or decision of a court or tribunal.

(A Concise Dict. of Law, ibid., p. 9.)

law : [...] a body of rules of action or conduct prescribed by controlling authority, and having binding legal force. (Black's Law Dict., 5th ed., West, 1978, p. 795.)

case : a court action; a legal dispute; the arguments, collectivity, put forward by either side in a court action. (A Concise Dict. of Law, ibid., p. 50.)

bar :

The presence, actual or constructive of the court.

(Black's Law Dict., ibid., p. 135.)

Muni de ces définitions, ayant dégagé les notions que ces termes recouvrent, le traducteur peut passer à la traduction, au transfert. Voici l'alternative qui s'offre à lui :

1. Traduire selon le principe du "transcodage ", ce qui donnerait à peu près ceci :

Un jugement implique l'application de la loi aux causes individuelles portées, d'une manière ou d'une autre, devant la barre de justice.

2. Traduire en interprétant le texte dans l'esprit de "l'équivalence fonctionnelle * (ou de la "transparence"). Cela pourrait donner, entre autres, la phrase suivante :

Rendre un jugement consiste à dire le droit dans un litige soumis à un tribunal.

Entre ces deux extrêmes, il est sans doute possible d'envisager une demi-douzaine de solutions acceptables ou satisfaisantes selon le contexte en cause. Ces deux exemples n'ont pour but que de montrer les ressources potentielles d'une langue, la française en l'occurrence, devant un problème de traduction donné et la nécessité de recourir à l'interprétation du texte pour en tirer une traduction satisfaisante du double point de vue, juridique et linguistique. Si le législateur désire vraiment que nul n'ignore la loi, objectif idéal de la forme la plus achevée de la démocratie, celle-ci doit être lisible et accessible au plus grand nombre. Il appartient aux juristes de donner l'exemple en cette matière et de conformer leur souci de précision avec un idéal de clarté et une volonté déterminée de l'inscrire dans les faits, faute de quoi, la " science [du droit] sans conscience " pourrait bien finir par être la "ruine de [leur] âme".

\section{CONCLUSION}

Le lecteur comprendra mieux pourquoi, dans ces conditions, il nous paraît utopique de prétendre vouloir former des traducteurs juridiques, polyvalents ou spécialisés, dans le cadre étroit d'un, voire de deux cours. Aussi préférons-nous employer le terme " jurilinguiste ", au sens où nous l'entendons depuis des années : au confluent du droit et de la langue, le traducteur (qui est aussi peu ou prou terminologue) juridique représente la synthèse de l'expression (linguistique) du droit. À ce titre, il dépasse la fonction de traducteur, il est aussi un interprète du droit, quoique d'un genre nouveau, à même d'apporter aux juristes quelque éclairage sur ce que ces derniers ont quelquefois tendance à considérer comme un domaine réservé, le langage du droit, qu'ils sécrètent, perpétuent et interprètent, quelquefois abusivement, et que le traducteur, par définition, a le devoir de replacer dans une perspective plus générale que particulière, peut-être même universelle, celle de la langue commune à tous.

Notes et renvois

* Texte reproduit avec l'autorisation de la Revue générale de droit d'Ottawa. (1987) : 18 R.G.D, 495-514.

1. Voir sur cette question Me Louis-Philippe Pigeon (1982) : " La traduction juridique - L'équivalence fonctionnelle", in Langage du droit et traduction, Québec, Linguatech, p. 271. 
2. Voir en particulier Jean-Claude Gémar : "La traduction juridique et son enseignement - aspects théoriques et pratiques", META, 24:1, p. 35 et suiv.; "La langue juridique, langue de spécialité au Québec ", The French Review, vol. LIII, no 6, mai 1980, p. 880 et suiv.

3. Bien que l'on puisse envisager diverses sortes de " contraintes", selon le texte considéré, par exemple dans le domaine religieux (contrainte morale), sur les plans social (mours), philosophique (dialectique), littéraire (l'esthétique), politique (selon les principes de gouvernement préconisés), etc. Voir aussi sur ce point : Peter Newmark : "The Translation of Authoritative Statements : A Discussion ", in Langage du droit et traduction, op. cit., p. 283 et suiv.

4. Pierre-André Côté (1982) : Interprétation des lois, Montréal, les Éditions Yvon Blais Inc., p. 10. Sur la dualité des méthodes d'interprétation en droit civil et en common law, voir pp. 9-14.

5. Â proportion du degré de développement d'un groupe social donné : le système juridique des sociétés dites avancées reflète la complexité, parfois inouie, de leur organisation. Ce trait caractérise la société ouverte, au sens que lui donne Carl Popper, par opposition à la société fermée.

6. Le phénomène de mimétisme entre langues latines (espagnol, français, italien...), comme entre le français et l'anglais, banalise souvent l'opération de transcodage au terme de laquelle le traducteur, devant les mots CONTRACT, CONTRATTO, CONTRATO (esp.), CONTRATO (port.), par exemple, traduire " naturellement " en français par CONTRAT. Autre chose sera de dire - et de savoir - ce que recouvre précisément ce mot dans chacun des systèmes considérés...

7. Voir, à ce sujet, Danica Seleskovitch : "Interprétation ou interprétariat ", META, 30:1, p. 19 et suiv.

8. Formule que le lecteur trouvera en annexe à la présente étude, car elle vient en conclusion à celle-ci et non en préalable, à titre d'illustration, en quelque sorte, des principes cardinaux que nous prônons en matière d'enseignement de la traduction en général et de la traduction juridique en particulier.

9. Par exemple, sur les travaux bien connus des jurilinguistes canadiens et les recherches effectuées en la matière par divers groupes et centres de recherche : à Ottawa (État fédéral), Québec, Moncton, Montréal, Toronto, etc.

10. Ce générique comprend l'avocat, le notaire, le juge, le professeur de droit, théoriciens et praticiens, et même les étudiants en droit.

11. Pour lire une opinion stimulante sur la question, par ex. : Steven Stark (1984) : "Why Lawyers can't Write ", Harvard Law Review, vol. 97, n⿳0 6, avril, p. 1389.

12. Voir en particulier : Michel Sparer et Wallace Schwab (1980) : Rédaction des lois, Québec, Conseil de la langue française, chap. 3, pp. 141-182; Michel Sparer : "La stéréophonie législative, facteur de haute infidélité " (1980) 21 C. de D. 599 (les Cahiers de droit, vol. 21, Presses de l'Université Laval, 1980, p. 599)

13. En common law, étant donné l'importance que l'on accorde à la jurisprudence, le rôle des juges a longtemps été, et reste encore dans une large mesure, prépondérant par rapport aux écrits de la doctrine, alors qu'en droit civil les techniques d'interprétation "font largement appel à la doctrine " (Côté : Interprétation des lois, op. cit., p. 496). Une telle habitude favorise assez peu l'expression claire et concise du droit, d'autant plus que les juges sont tous d'anciens avocats et en ont conservé le style (et l'inflation verbale) dans les jugements qu'ils rédigent, le plus souvent, à la manière d'une plaidoirie, donc de façon personnalisée plutôt qu'impersonnelle. Toutefois, là aussi les choses changent et les mentalités évoluent : "[...] les tribunaux paraissent réserver une plus large place dans leurs jugements à l'opinion doctrinale " (Côté : ibid., p. 496). La question de la rédaction est traitée en détail dans notre étude : les Trois états de la politique linguistique du Québec, Conseil de la langue française, 1983.

14. Un arrêt est un jugement rendu par un tribunal de juridiction " supérieure " (cour d'appel et au-dessus). Cf. Dictionnaire du droit privé, Montréal, Centre de recherche en droit privé et comparé du Québec, 1985, Vo arrêt, p. 21.

15. Bien qu'à notre avis, il soit préférable de commencer par la formation linguistique avant d'entreprendre une quelconque spécialisation, juridique ou autre. Le contraire semblerait donner de moins bons résultats, d'après notre propre expérience fondée sur une quinzaine de générations d'étudiants des premier et deuxième cycles.

16. Nous reprenons ici les termes juridiques définis dans le Dictionnaire de droit privé (op. cit., p. 135) en leur donnant aussi le sens que nous appliquons à diverses situations de traduction.

17. En général, mais nous visons ici plus particulièrement celui qui travaille dans le domaine juridique.

18. Voir ce mot dans le Dictionnaire de droit privé, op. cit., p. 29.

19. Loi sur la protection des renseignements personnels, S.C. 1980-81-82-83, chap. 111, annexe II.

20. Voir à ce sujet : A.-F. Bisson (1980) : "L'interaction des techniques de rédaction et des techniques d'interprétation des lois ", 21 C. de D. 511.

21. Il convient de nuancer cette affirmation selon le genre de textes envisagés dans cette catégorie. Toutefois, en règle général, on invoque les impératifs de temps, de la production, de la concurrence du marché, les besoins du client, etc.

22. Sur cette question : voir Lisette Savard, "Introduction au droit : des étudiants insatisfaits ", Barreau 84, vol. 16-11, Québec. Voir aussi la critique de l'enseignement du droit actuellement dispensé aux États-Unis 
qu'a faite le professeur Bok, doyen de l'École de droit de Haryard. Un tel enseignement a pour effet d'entrainer "a massive diversion of exceptional talent into pursuits that often add little to the growth of the economy, the pursuit of culture or the enhancement of the human spirit ". Le doyen Bok recommande aux professeurs de droit de faire davantage appel aux techniques d'analyse utilisées en sciences sociales, qu'il qualifie de "steadily more refined" (Time, May 2, 1983, pp. 80-81).

23. Ce qui n'exclut nullement qu'elle puisse aussi être "pleine", quoique cela ne constitue pas l'objectif premier de notre enseignement.

24. Par ex., des contrats : baux, assurances, vente ; en droit du travail : conventions collectives ; en procédure civile ou pénale ; en droit administratif : communications, énergie et ressources; en droit commercial : sociétés, effets de commerce, faillite; etc.

25. Un exemple illustrera ce principe : comprendre, à la lecture d'un mot, que CONTRACT $=$ CONTRAT relève d'une connaissance minimale des deux langues en présence. Le spécialiste, en revanche, saura les divers sens possibles de ces mots dans chaque langue et comment les utiliser, les reconnaître dans un texte. De ce point de vue, il saura "prendre la température " du texte, qu'il interprétera d'autant plus aisément et correctement.

26. De toutes les langues de spécialité, la langue juridique est peut-être celle où règne la plus grande polysémie.

27. Science et technique en droit privé positif, Paris, Sirey, 1921, t. 3, no 255 , p. 456. Cette terminologie, parlons-en, est souvent mal connue, mal comprise et mal utilisée par nombre de juristes (et la majorité des gens), dans la plupart des langues, des systèmes et des pays. Les difficultés qu'éprouvent les juristes nordaméricains en la matière sont proverbiales. Au Québec, elles sont dues à des causes historiques et au caractère hybride du droit qui ont engendré une situation de diglossie. Le nombre ne saurait à lui seul tout expliquer, bien que les chiffres suivants aient de quoi donner le vertige : pour le seul droit privé du Québec (et du Canada, applicable au Québec), le corpus traité pour établir le Dictionnaire de droit privé dépasse 10000 termes. Ce nombre doit être multiplié par 3 ou 4 si l'on veut se rapprocher de la vérité et trouver le nombre total - approximatif - de termes que contient la langue juridique française. Un tel volume pourrait expliquer les insuffisances des juristes et leurs difficultés à maîtriser un vocabulaire d'une telle richesse. Cette raison ne résiste toutefois pas à l'analyse : un juriste connaît ipso facto le vocabulaire de sa spécialité, qui lui a été enseigné dans les facultés de droit ; il l'utilise dans son travail quotidiennement, couramment sinon facilement. Est-ce qu'il le possède pour autant? Il faut en chercher les vraies raisons ailleurs, ce qui n'est pas l'objet de notre propos. Voir, par ex. : les Trois états de la politique linguistique du Québec, op. cit.

28. Une expérience de traduction originale a été tentée - avec succèes - à l'Université de Montréal voici plusieurs années : un étudiant s'est efforcé de traduire en français fondamental un texte technique sur le plancton (domaines : chimie et biologie). Cela a donné une traduction considérablement "délayée "et beaucoup plus longue qu'elle ne l'aurait été si l'on avait employé les termes techniques requis.

29. Dont l'auteur de cette étude a eu le privilège de faire partie du comité de rédaction.

30. Au contraire, nous croyons à l'interdépendance du fond et de la forme, inextricablement liés et non indépendants l'un de l'autre, comme certains voudraient le faire croire, au premier rang desquels les juristes, qui distinguent très souvent le «fond" de la «forme ». Nous croyons que la forme (vocabulaire, syntaxe, expression ou style) est, à sa façon, porteuse de sens au même titre que le contenu, le message. Bien que ce ne soit pas le lieu de s'en expliquer longuement ici, ce principe n'en guide pas moins notre action pédagogique, quel que soit le degré de " pragmatisme " du texte - juridique ou autre - concerné. $\mathrm{La}$ façon de dire n'est jamais innocente ni fortuite et, à cet égard, elle est aussi un véhicule par lequel s'exprime le sens.

31. Cf. A Concise Dictionary of Law (1983), Oxford University Press, Vo term (2. Any provision forming part of a contract) et condition (1. A major term of a contract).

ANNEXE 1

Présentation d'un cours type de traduction juridique

Posons les questions suivantes :

1. À QUI enseigner : le public cible

Prenons le plus grand dénominateur commun, l'étudiant de $1^{\text {er }}$ cycle universitaire. C'est en fonction de cet étudiant type et de ses besoins (à établir : traducteur de 
lois, règlements, jugements ; ou d'actes juridiques ; de textes de doctrine) que l'on fixera les objectifs à atteindre et la méthode à suivre.

Par ex. : soit un groupe de 25 (ou moins, de préférence) étudiants + un cours d'introduction à la traduction juridique.

N.B. : On pourrait aussi envisager les cours suivants : initiation, perfectionnement, renforcement, spécialisation, révision, rédaction, interprétation, etc.

\section{QUOI enseigner : le contenu du cours}

Prenons encore un cours universitaire type : un trimestre, soit 12-13 séances de 3 heures chacune.

Ce cours peut être : 1) unique ; 2) le premier d'une série de 2, 3 ou 4.

Selon le cas, il s'agira :

1. de faire un tour d'horizon du droit, de son système, vocabulaire, etc., en 8 ou 10 volets ouverts sur les principaux domaines qui le composent;

2. de scinder cette présentation afin de la répartir et de l'approfondir (au besoin) en fonction des cours à venir.

Dans le premier cas, le contenu portera sur :

- les systèmes juridiques en présence

- leur vocabulaire

- leurs structures

- leur fonctionnement

Ce contenu pourrait être ainsi réparti sur 10-11 volets :

- les sources des systèmes juridiques ( 2 volets)

- les fondements constitutionnels (1 volet)

- les structures administratives (1 volet)

- l'organisation judiciaire et son fonctionnement (1 volet)

- le droit privé : common law. et droit civil (2 volets)

- le droit commercial (1 volet)

- le droit pénal (1 volet)

le droit international public/privé (1 volet chacun)

Ce tableau peut être brossé de nombreuses façons différentes selon les besoins. Voici, par ex. deux formules de présentation possibles :

1. - les droits de la personne

- la famille

- les biens

- les contrats

- la responsabilité civile

2. - introduction à l'étude du droit

- la méthodologie juridique

- droit public

- droit privé

- l'organisation judiciaire
- le droit du travail

- le droit des affaires

- les successions

- le système judiciaire

- le langage du droit

- les obligations

- la responsabilité (civile/pénale)

- droit pénal général

- droit commercial

- droit du travail

\section{COMMENT enseigner - la méthodologie}

Trois grandes formules sont possibles :

1. Faire un cours de droit (et ignorer la traduction, ses méthodes, contraintes...) ;

2. Faire de la traduction uniquement (et laisser les étudiants se débrouiller avec le "juridique»); 
3. Faire un panachage de présentations, d'explications et d'exercices de traduction appliquée.

La troisième formule, la plus complexe à organiser et à mettre en œuvre, nous semble la plus appropriée et répond aux besoins spécifiques du traducteur. Examinons la plus en détail :

1. Une formule mixte répondant à des besoins particuliers :

- alternance de présentations et d'explications ponctuelles : exposés du professeur sur la matière + lectures préparatoires et postérieures obligatoires;

- exercices de traduction préparés, en application des exposés, sur un sujet précis ;

- orientation terminologique sur les notions, termes et familles de termes fondamentaux du domaine étudié; le tout assorti de recherches documentaires et d'orientation bibliographique.

2. L'esprit de la méthode ou les fondements d'une jurilinguistique -enseigner le droit comme à des futurs juristes

-de façon comparative ( 2 systèmes au moins)

- en applicant les méthodes et principes de l'enseignement de la traduction générale (aspects linguistiques, traitement terminologique, utilisation des outils documentaires...)

- orientation terminologique sur les effets éventuels de la traduction de textes juridiques (l'interprétation des deux textes par un juge et ses conséquences juridiques) et les principes de rédaction dans chacune des langues en présence

Résumons dans un tableau la formule chiffrée de déroulement d'un cours type de traduction juridique.

1. Orientation, présentation, mise en train, etc.

2. Exposé du professeur sur la matière du jour

Temps consacré à chaque activité (en minutes)

3. Questions de la classe et discussion sur la matière présentée et les lectures obligatoires
$\leqslant 15^{\prime}$
$\geqslant 30^{\prime}$
$\leqslant 30$ '
$\geqslant 60^{\circ}$
$\geqslant 15^{\prime}$
$\leqslant 5$,

4. Pause

5. Exercices de traduction et applications de textes

d'application

6. Orientation terminologique

7. Orientation documentaire (recherches et lectures)

Total $=$

$\pm 170^{\prime}$ 
N.B. : Ces chiffres sont donnés à titre indicatif uniquement. Leur somme ne représente que 170' (sur 180' d'un cours " normal " de 3 heures) pour tenir compte des variables, par ex. : correction d'un examen, exposé plus ou moins long, questions plus ou moins nombreuses, matière plus ou moins vite comprise, explications plus ou moins longues, etc.

\section{ANNEXE 2 \\ Code de déontologie du traducteur juridique}

Article $1^{\text {er }}$ :

Article 2 :

Article 3 :

Article 4 :

Article 5:

Article 6:

Article 7 :

Article 8 :

Article 9 :

Article 10 :
Généraliste du droit, de préférence à spécialiste, de devenir t'efforceras.

Traducteur avant tout resteras, car de la langue au service demeureras.

Les principes généraux du droit parfaitement assimileras.

Le langage du droit et ses subtilités possèderas.

La langue d'arrivée - ta langue - maîtriseras.

Le système juridique étranger et la langue qui l'exprime le mieux possible comprendras.

Le système juridique d'arrivée sous tous les angles connaîtras.

L'auteur du texte de départ et son intention, de même que son expression le moins possible trahiras.

Ta langue et son style propre respecteras. À la facilité jamais ne céderas et de rigueur preuve tu feras. 